\title{
Study of GaAs/Fe Core/Shell Nanowires using Electron Holography
}

\author{
D. Zhang ${ }^{1}$, R. E. Pimpinella ${ }^{2}$ X. Liu ${ }^{2}$, M. Dobrowolska ${ }^{2}$, J. K. Furdyna ${ }^{2}$, D. J. Smith ${ }^{3}$ and \\ M. R. McCartney ${ }^{3}$ \\ 1. School of Engineering for Matter, Transport, and Energy, Arizona State University, Tempe, AZ 85287 \\ 2. Department of Physics, University of Notre Dame, Notre Dame, IN 46556 \\ 3. Department of Physics, Arizona State University, Tempe AZ 85287
}

Magnetic structure at the nanometer scale has attracted considerable interest due to its significance from the viewpoints of both fundamental science and practical applications. Magnetic nanoscale "tubes" are one of these new structures and core-shell GaAs/Fe nanowires (NWs) are representative of such structures. The GaAs NWs studied here were grown by the VLS method using molecular beam epitaxy (MBE), followed by Fe deposition in order to create thin Fe shells on the GaAs cores in the form of Fe "nanotubes". The shells were finally coated with Au to prevent Fe oxidation [1]. NWs for microscopic examination were prepared by dispersion in isopropanol using an ultrasonic bath for 3 min, followed by spreading a drop from the suspension on a 200 mesh uniform carbon/ $\mathrm{Cu}$ grid. High-resolution images were recorded with a JEM-4000EX HREM at $400-\mathrm{keV}$ with a structural resolution of $\sim 1.7 \AA$. STEM images, EDXS and EELS spectra was recorded by JEM-2010F with EDAX X-Ray acquisition system and Enfina EELS detector. The magnetic structure was studied by off-axis electron holography technique using an FEI CM-200 equipped with electrostatic biprism and Lorentz lens.

The GaAs NWs grow on (111)B zincblende GaAs with growth axis normal to the substrate. The NWs form with the wurtzite structure but also contain many stacking faults [Fig. 1(a)]. However, the Fe shell does not grow as an epitaxial layer and metal clusters are also visible. Lattice fringes within the clusters indicate Au spacing [Fig. 1(b)]. HR-STEM images recorded from one of these NWs reveal Au clusters on the surface and incomplete Au coating [Fig. 2(a)]. EDXS line scans indicate the presence of iron and oxygen in the shell [Fig. 2(b)]. After saturating the magnetization of the NWs in certain directions, electron holograms were recorded using the Lorentz lens in a field-free condition [2]. As well as NWs grown on (111)B substrates, NWs grown on (110) substrates were also studied. Due to the preferred NW growth direction, these NWs have an inclination of $\sim 35.3^{\circ}$ [3] with respect to the substrate, resulting in rotationally non-uniform coating. Reconstructed phase maps show no fringing magnetic field around NWs grown on (111)B substrates [Fig 3(a)]. On the other hand, a phase jump of about 3rad is visible across NWs grown on (110) substrates [Fig 3(b)]. Based on the present results, it can be concluded that NWs grown on (111)B substrates have a cylindrical shell with a magnetic field confined within the shell and forming closed loops. Using EELS, the presence of iron can be shown and the ratio of $\mathrm{Fe} / \mathrm{O}$ can be deduced. These results will also be reported at the meeting [4].

\section{References:}

[1] K. Tivakornsasithorn, et al, J. Vac. Sci. Technol B. 30 (2012) 2.

[2] M. R. McCartney, D. J. Smith, Ann. Rev. Mater. Res. 37 (2007) 729.

[3] R. E. Pimpinella, et al, J. Appl. Phys. in press.

[4] This work was supported by DOE Grant DE FG02-04 ER46168. We gratefully acknowledge the use of facilities within the LeRoy Eyring Center for Solid State Science at Arizona State University, and we thank Dr. T. Aoki for his assistance with microscope adjustment. 

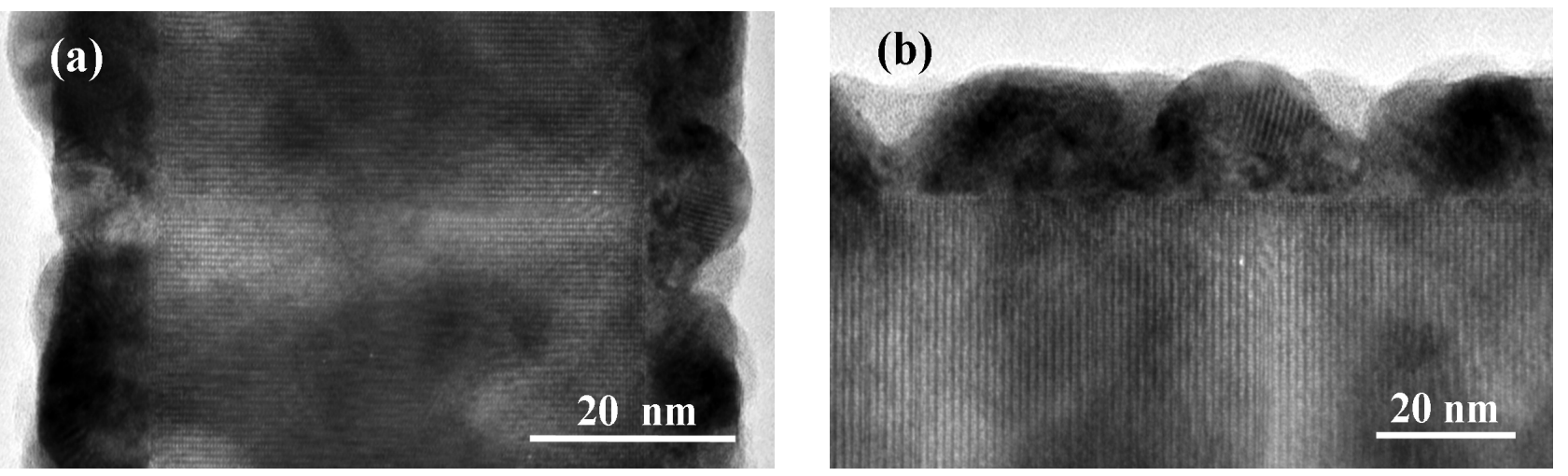

Figure 1.(a) High-resolution TEM images showing GaAs core, and (b) Au/Fe shell, of GaAs/Fe/Au NW.
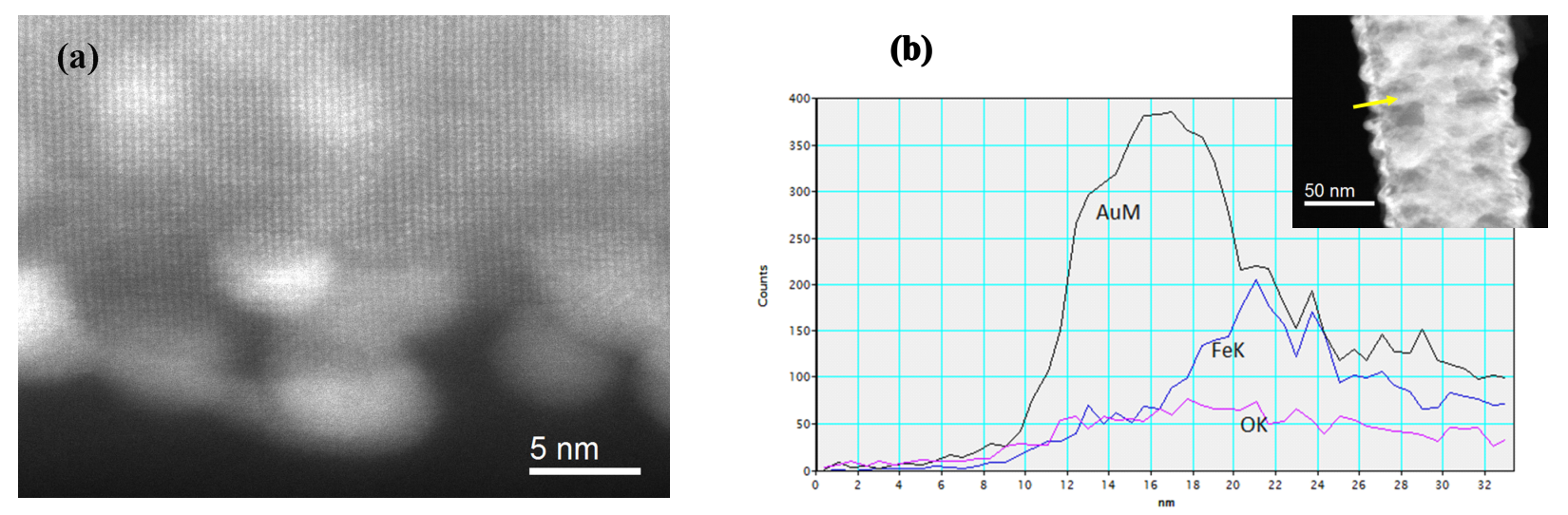

Figure 2. (a) High-resolution STEM image of Au clusters on GaAs surface, and (b) EDXS line profile from position indicated in insert.
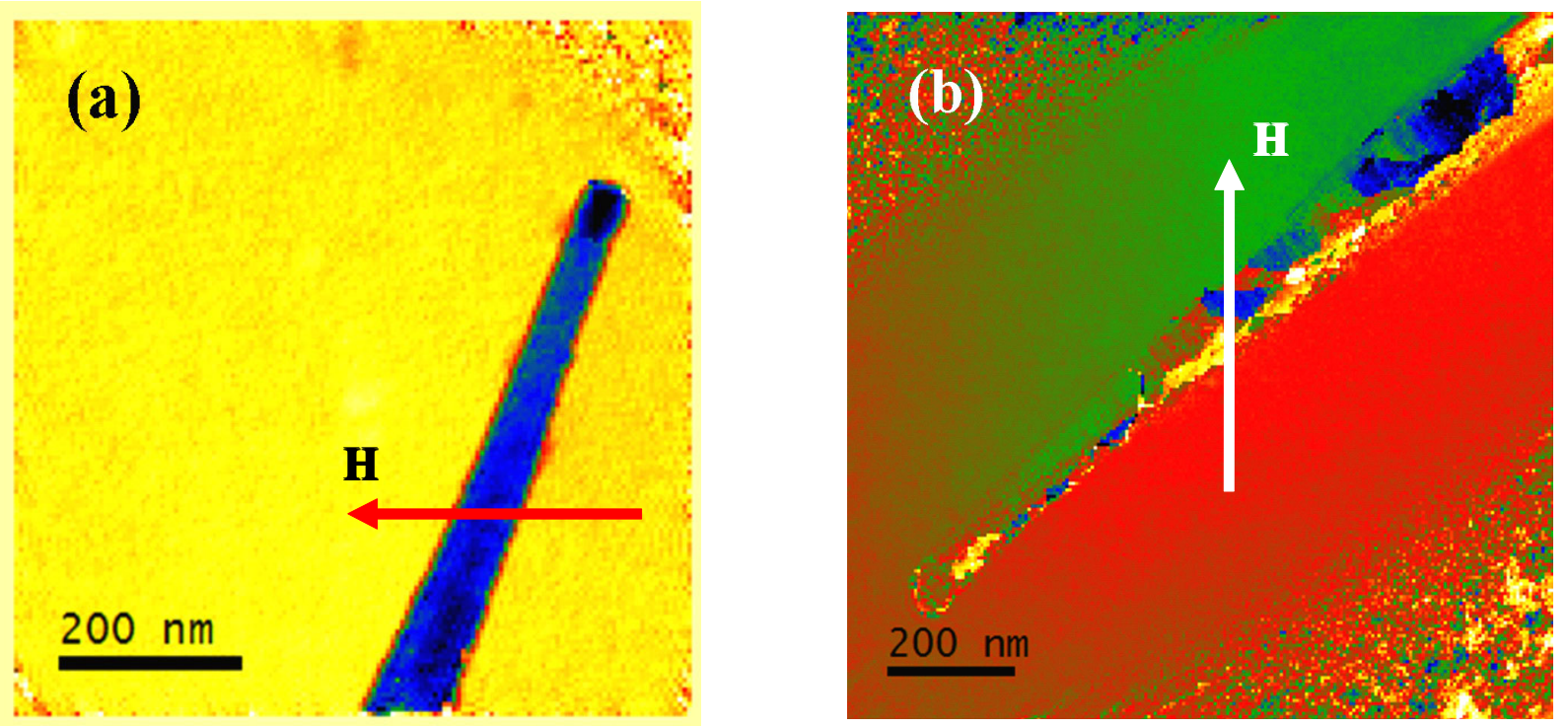

Figure 3. (a) and (b): Reconstructed phase maps of NWs grown on (111)B, and (110) substrates, respectively. The arrows indicate directions of the applied magnetic field. 